\title{
Psychosis and relapse in bipolar disorder are related to GRM3, DAOA, and GRIN2B genotype
}

\author{
S Dalvie', N Horn², C Nossek', L van der Merwe ${ }^{3,4}$, DJ Stein², R Ramesar ${ }^{1}$ \\ 1Division of Human Genetics, MRC/UCT Human Genetics Research Unit, Faculty of Health Sciences, University of Cape Town Medical \\ School, Observatory, Cape Town, South Africa \\ 2Department of Psychiatry and Mental Health, University of Cape Town Medical School, Observatory, Cape Town, South Africa \\ 3Biostatistics Unit, Medical Research Council, Cape Town, South Africa \\ ${ }^{4}$ Department of Statistics, University of Western Cape, Bellville, South Africa
}

\begin{abstract}
Objective: Dysfunction in glutamate signalling is thought to play a role in the pathophysiology of bipolar disorder (BD). There is evidence of associations between single nucleotide polymorphisms (SNPs) in GRM3, GRIN2B, and DAOA genes and the diagnosis of BD. In this pilot study, we investigated the frequency of SNP variants in these 3 genes within South African population groups, and assessed interactions between genes and phenotypes of BD disease severity. Method: Multiplex SNaPshotTM PCR was used to genotype 191 case and 188 control samples. Cases comprised of 191 individuals in a South African cohort of mixed ancestry and Caucasians, with BD Type 1. Phenotypes of BD disease severity were: age of onset, number of illness episodes, number of hospitalisations for depression or mania and history of psychotic symptoms. Results: There were no significant difference in SNP allele frequencies between cases and controls. In the case-only analysis, the GRM3 rs6465084 heterozygote was associated with a 4-fold increased risk of lifetime history of psychotic symptoms, and the specific variants within the gene pair, DAOA and GRIN2B, had a significant interaction with the number of hospitalisations for mania, with lowest admission rates associated with both pairs of ancestral alleles. Conclusion: In BD, variations in glutamatergic genes may influence phenotypes related to the severity of illness. Speculatively, newly derived genes associated with various evolutionary advantages, may also increase the risk for more severe BD. These preliminary findings deserve validation in a larger cohort.
\end{abstract}

Key Words: Manic-Depressive Psychosis; Glutamate; GRIN2B receptor; mGluR3; G72 protein; Human

Received: $20-04-2010$

Accepted: 02-08-2010

\section{Introduction}

The lifetime prevalence of bipolar disorder Type 1 (BD) is approximately 1\% in the US and Europe, and likely equally high in Southern Africa. ${ }^{1,2}$ BD is associated with a high level of mortality (between 10-20\% of individuals affected with BD successfully commit suicide) and morbidity (BD is estimated as one of the top ten causes of disability worldwide). ${ }^{3-5}$ An earlier age of disease onset, increased number of episodes and hospitalisations for mania and depression, and the presence of psychotic symptoms are markers of a more severe disease phenotype. ${ }^{6-8}$ Previous research has not examined associations

Correspondence
Professor Rajkumar Ramesar
Division of Human Genetics, Faculty of Health Sciences,
University of Cape Town Medical School, Observatory, Cape Town,
South Africa, 7925
email: Raj.Ramesar@uct.ac.za

between phenotype severity and glutamate signalling in BD, although variants within brain derived neurotrophic factor (BDNF) may be associated with indices of severity in BD.9-11

Glutamate signalling is involved in brain development and synaptic plasticity, both of which are modified in individuals affected with $\mathrm{BD}$, and have been implicated in its aetiology.12,13 A range of psychotropics involved in the treatment of BD alter glutamatergic function; for example, lamotrigine inhibits the release of glutamate and lithium affects glutamate receptor function. ${ }^{14}$ It has also been observed that variation in the glutamate receptor encoding gene, GRIK4 (rs 1954787) makes subjects with depressive episodes more likely to respond to citalopram. ${ }^{15}$ Fallin et al. found that the glutamatergic genes most likely to be associated with BD rather than schizophrenia (SZ) were GRM3 and GRIN2B, and replicated previous findings of strong associations between DAOA and BD diagnosis. ${ }^{16}$

Glutamate receptor, metabotropic 3 (GRM3) encodes subunit 3 of the Group II metabotropic glutamate receptors 
(mGluR). In glutamate receptor, metabotropic 2 (GRM2) and GRM3 knockout mice, Lyon et al. showed a compensatory upregulation of remaining group II mGluRs and the N-methyl D-aspartate (NMDA) receptor subunit 2A (NR2A) expression, and down-regulation of glutamate transporter expression. ${ }^{17}$ Post-mortem studies have suggest altered GRM3 expression in BD. ${ }^{18}$ Crespi et al. identified GRM3 as one of 14 genes with significant evidence of selection in human populations. ${ }^{19}$ GRM3 modulates glutamate neurotransmission by regulating the excitatory amino acid transporter 2 (EEAT2). Egan et al. have demonstrated that decreased levels of EEAT2 are associated with a GRM3 SNP (rs6465084), which has a weak association with SZ. ${ }^{20}$ Egan et al. have also demonstrated that individuals who are homozygous A for GRM3 rs6465084 have lower levels of $\mathrm{N}$-acetylaspartate, an indirect indicator of synaptic function and glutamate levels and suggests that GRM3 rs6465084 has an effect on GRM3 transcript expression. ${ }^{20}$

Ionotropic glutamate receptor, $\mathrm{N}$-methyl $\mathrm{D}$-aspartate $2 \mathrm{~B}$ (GRIN2B) encodes the NR2B subunit of the NMDA receptor. Mice with enhanced NR2B function show supernormal learning and memory function, while BD sufferers consistently show impairment in working memory. ${ }^{21}$ Associations have been observed between several polymorphisms in the GRIN2B gene and the 12p13.1-pl2.3 region (containing the GRIN2B gene) and BD. 16,22,23 Miyatake et al. have observed that the $\mathrm{T}$ allele of the GRIN2B rs1019385 SNP results in increased luciferase transporter activity in the presence of nerve growth factor (NGF). ${ }^{24}$ The presence of a $G$ allele had no effect on reporter activity, suggesting that GRIN2B rs1019385 may have a role in transcriptional control of the GRIN2B transcript. ${ }^{24}$

The G72 gene product, D-amino-acid oxidase activator (DAOA), activates the peroxisomal protein $\mathrm{D}$-amino-acid oxidase (DAO). ${ }^{25}$ This protein (G30) degrades D-serine, which acts, similar to glycine, as a coactivator on the "glycine binding site" of the glutamatergic NMDA receptor. Hashimoto et al. showed that the percentage of D-serine in the total serine in the cerebrospinal fluid of drug naive SZ patients was significantly lower than that of control samples. ${ }^{26}$ Genetic variants of G72/G30 have been found to be associated with BD and SZ. ${ }^{16,27-32}$ In some populations, the at-risk haplotypes are shared between SZ and BD. ${ }^{31}$ The pathogenic mutations have not yet been identified but might be located in the vicinity of the G72/G30 gene complex or in the regulatory region. ${ }^{30}$

\section{Method}

\section{Subjects}

The cohort comprised of two groups: a group of BD Type 1 affected individuals (cases) and the background population group (controls). Blood samples were previously obtained, with the appropriate written informed consent. This study was approved by the University of Cape Town Health Science Faculty Research Ethics Committee (081/96) and is in accordance with the guidelines of the Helsinki Declaration of 2000.

The Division of Human Genetics has a BD database consisting of 883 individuals comprising 185 families. For the purposes of this study, the cases consisted of 191 individuals specifically diagnosed with BD Type 1 with the Structured Clinical Interview for the DSM-IV (SCID). This group comprised of 87 (46\%) males and 104 (54\%) females of ages ranging from 22 to 91 years. Subjects included 103 (54\%) individuals of mixed ancestry and 88 (46\%) Caucasian individuals. The cases included 95 individuals from 36 families that were related to at least one other case. The age of onset, number of depressive episodes, number of hospitalisations due to mania and depression of each of the cases were noted, as well as history of psychotic symptoms. The control group consisted of 188 unrelated individuals not diagnosed with any psychiatric disorder, and similar to the cases in terms of age, gender and ethnicity.

\section{DNA Analysis}

The following SNPs were chosen for genotyping based on previous association to either BD or SZ: GRM3 rs6465084 (intronic), GRIN2B rs1019385 (5' upstream), and DAOA rs701567 (intronic). ${ }^{1-4}$ Gene sequences were obtained from the National Center for Biotechnology Information (NCBI) (http://www.ncbi.nlm.nih.gov) and Ensembl

(http://www.ensembl.org/index.html) databases and the SNP locations were determined. DNA concentration and integrity was determined by spectrophotometric quantification and agarose gel electrophoresis, respectively. Polymerase chain reaction (PCR) was performed on genomic DNA, according to standard procedures, for each of the SNPs in the candidate genes. The polymorphisms were genotyped using the SNaPshot ${ }^{\mathrm{TM}}$ multiplex ready reaction mix (Applied Biosystems, Warrington, UK) and the ABI Prism 3100 Genetic Analyzer (Applied Biosystems, CA, USA). Genotyping results were validated by cycle sequencing using BigDye Terminator Mix (Applied Biosystems, CA, USA) and the ABI Prism 3100 Genetic Analyzer (Applied Biosystems, CA, USA).

\section{Statistical Analysis}

For the case-control analysis, one individual from each family was randomly selected, leaving 132 unrelated cases. For this analysis, logistic regression was used.

The case-only analyses involved all 191 cases, which were adjusted for relatedness. We used generalised linear models for all the association tests. For age of onset, we used a normal family with an identity link; for each of the three outcomes: (i) number of depressive episodes, (ii) hospitalisations due to mania and (iii) hospitalisations due to depression, we used the Poisson family with a log link and for dichotomous history of psychosis, a binomial family with logit link. There were only 4 GRM3 rs6465084 homozygous G cases. We therefore grouped them with the heterozygotes for the case-only analyses. Genotypes were coded as categories for the single-SNP analyses, and numerical (number of minor alleles) for testing interactions.

All of the six outcomes analysed (case-control, age of onset, number of depressive episodes, hospitalisations due to mania, hospitalisations due to depression, and history of psychotic events), were tested for an interaction between the three SNPs. When this was not significant, we tested all pairwise interactions between SNPs and where those were not significant, we tested the individual SNPs (main effects). All analyses were adjusted for gender, ethnicity and duration of disease as fixed effects and family-membership as random effect.

Statistical analyses were done with $\mathrm{R}$, a language and environment for statistical computing and graphics, and $\mathrm{R}$ packages nlme and MASS (http:Ilwww.R-project.org). 


\section{Results}

Case-control analysis (132 cases; 188 controls)

The differences in the genotype and allele frequencies between cases and controls were not significant. Neither were any SNP-interactions on case-control status significant (results not shown). The allele frequencies for each of the SNPs obtained from the cases and controls in the current study, are displayed in Table I.

\section{Case-only analysis $(n=191)$}

All the results presented here were adjusted for gender, ethnic group, duration of disease and family-membership.

We did not find any significant association between the SNPs GRM3 rs6465084, GRIN2B rs 1019385, DAOA rs701567 and main effects or interactions on age of onset ( $n=124)$, number of depressive episodes $(n=130)$, or number of hospitalisations for depression $(\mathrm{n}=171)$. However, a significant association between GRM3 rs6465084 and history of psychosis (63\% with a history and 37\% without history of psychosis) was found. The GRM3 rs6465084 G-allele was found to increase the odds of having had a psychotic episode $(\mathrm{OR}=3.9 ; \mathrm{p}=0.004)$ considerably. Even after adjusting for family-relatedness only, a significant association $(\mathrm{OR}=2$.2; $\mathrm{p}=0.0137$ ) was observed.

Reported number of hospitalisations for mania ( $\mathrm{n}=167$ ) varied between 0 and 11 . The interaction between $\mathrm{DAOA}$ rs701567and GRIN2B rs 1019385 on number of hospitalisations for mania was significant ( $p=0.0108)$, and this interaction was independently significant after including other interactions in the model. The nature of the modelled interaction is illustrated in Figure 1 and can be described as follows: For individuals with the GRIN2B rs 1019385 genotype G/G, their expected number of hospitalisations for mania increased with their number of DAOA rs701567 G alleles. For individuals with the GRIN2B rs 1019385 genotype G/T, there were smaller decreases in number of hospitalizations and for those individuals with the T/T genotype, each DAOA rs701567 G allele resulted in larger decreases. Individuals who are homozygous A for DAOA rs701567 and homozygous T for GRIN2B rs 1019385 had the largest number of hospitalisations, and those who are homozygous $G$ for DAOA rs701567 in combination with being homozygous G for GRIN2B rs 1019385 , had the second largest. Those with DAOA rs701567 G/G and GRIN2B rs 1019385 T/T had the least number of hospitalisations.

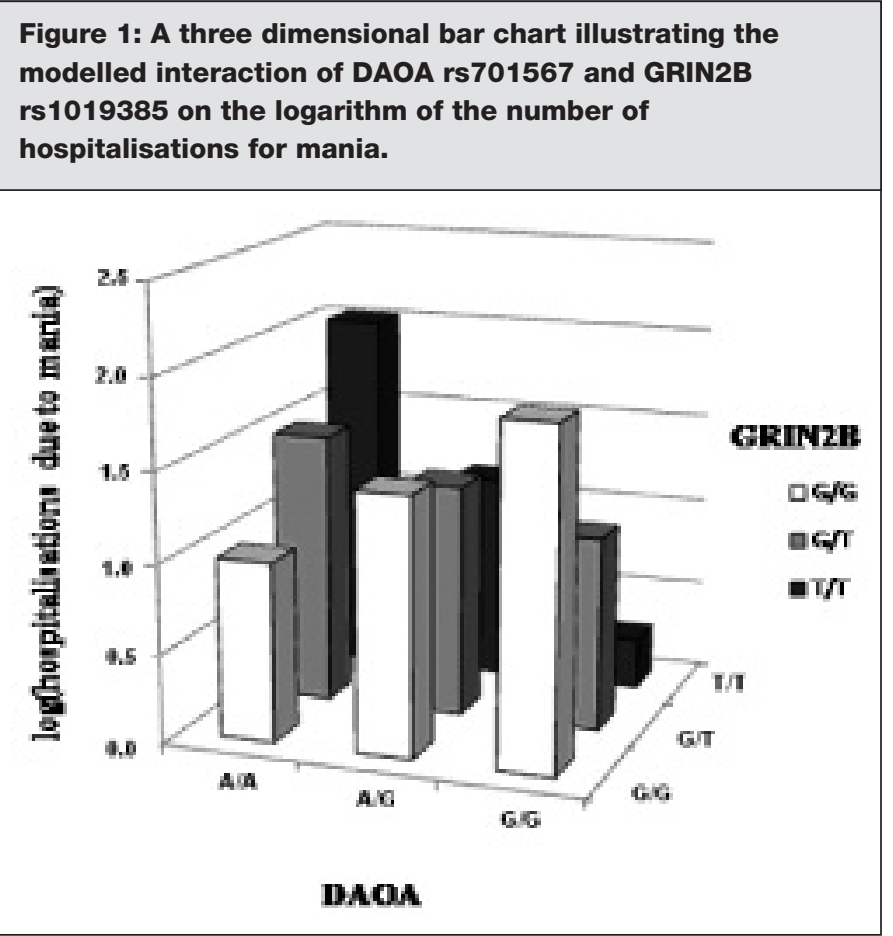

\section{Discussion}

The first set of findings related to SNP frequency showed that all 3 SNPs examined had a similar distribution between Caucasian and mixed ancestry groups, and that BD diagnosis was not associated with any of the SNPs examined. The second set of findings, related to BD severity, was that the nonancestral GRM3 G allele at rs6465084 carries a 4 times greater risk of developing psychosis in $\mathrm{BD}$ subjects, and that alleles at DAOA rs701567 and GRIN2B rs 1019385 interact such that the risk of hospital admission is higher with a greater number of non-ancestral alleles.

This is the first time that allele frequencies in the candidate genes studies here have been described for the mixed ancestry population of South Africa. SNP frequencies were broadly similar to reported frequencies for Caucasian groups described elsewhere (NCBI http://www.ncbi.nlm.nih.gov). ${ }^{22}$ No difference in frequency was detected for the ancestral allele for GRM3 rs6465084 and of GRIN2B rs 1019385 in individuals of both Caucasian and mixed ancestry. The A/G alleles of DAOA rs701567 were found to occur at almost equal

Table I: SNP Allele Frequencies For Each of the Candidate Genes

\begin{tabular}{|c|c|c|c|c|c|c|c|}
\hline Gene & SNP & Ethnic Group & No. of Cases & No. of Controls & $\begin{array}{l}\text { Ancestral Allele } \\
\text { Frequency Cases }\end{array}$ & $\begin{array}{l}\text { Ancestral Allele } \\
\text { Frequency Controls }\end{array}$ & $p$-value \\
\hline GRM3 & rs6465084 & $\begin{array}{l}\text { Caucasian, Mixed Ancestry } \\
\text { Caucasian only } \\
\text { Mixed Ancestry only }\end{array}$ & $\begin{array}{l}132 \\
69 \\
63\end{array}$ & $\begin{array}{l}188 \\
89 \\
99\end{array}$ & $\begin{array}{l}0.75 \\
0.73 \\
0.77\end{array}$ & $\begin{array}{l}0.77 \\
0.78 \\
0.76\end{array}$ & $\begin{array}{l}0.5953 \\
0.3054 \\
0.7887\end{array}$ \\
\hline GRIN2B & rs1019385 & $\begin{array}{l}\text { Caucasian, Mixed Ancestry } \\
\text { Caucasian only } \\
\text { Mixed Ancestry only }\end{array}$ & $\begin{array}{l}132 \\
69 \\
63\end{array}$ & $\begin{array}{l}188 \\
89 \\
99\end{array}$ & $\begin{array}{l}0.35 \\
0.41 \\
0.29\end{array}$ & $\begin{array}{l}0.34 \\
0.40 \\
0.28\end{array}$ & $\begin{array}{l}0.8268 \\
0.9181 \\
0.8304\end{array}$ \\
\hline DAOA & rs701567 & $\begin{array}{l}\text { Caucasian, Mixed Ancestry } \\
\text { Caucasian only } \\
\text { Mixed Ancestry only }\end{array}$ & $\begin{array}{l}132 \\
69 \\
63\end{array}$ & $\begin{array}{l}188 \\
89 \\
99\end{array}$ & $\begin{array}{l}0.43 \\
0.41 \\
0.45\end{array}$ & $\begin{array}{l}0.48 \\
0.41 \\
0.56\end{array}$ & $\begin{array}{l}0.2000 \\
0.8961 \\
0.0725\end{array}$ \\
\hline
\end{tabular}


frequencies in both population groups.

We did not demonstrate that any of the putative risk alleles were associated with BD diagnosis. This is consistent with the lack of consistently reproducible associations between individual genes and BD diagnosis even in large data sets. ${ }^{33}$ There is increasing evidence that $\mathrm{BD}$ is a disorder where many genes of small effect contribute to risk of developing the disorder. ${ }^{20}$

In this study, we observed that the $\mathrm{G}$ allele at SNP rs6465084 GRM3 carries a 4 times greater risk of developing psychosis in subjects with BD. This is unexpected as the A allele in this SNP has been weakly associated with SZ diagnosis- and might be expected to be a risk factor for psychosis in BD. ${ }^{20}$ This finding by Egan et al., however, was not replicated by others. ${ }^{34,35}$ Egan et al. also reported an association between the A allele, impaired list learning and verbal fluency, and low prefrontal levels of glutamate transporter EEAT2 in normal subjects and people with SZ. ${ }^{20}$ It is possible to speculate that the A allele confers a risk of a dysexecutive syndrome typical of certain types of SZ, while the $G$ allele is associated with normal or enhanced executive function, consistent with the finding by Diller et al. that GRM3 is a gene which has evolved favourable new (derived) alleles. ${ }^{36}$ The $\mathrm{G}$ allele may, however, together with other risk factors, be associated with more severe mania. Crespi et al. identified GRM3 as a gene with significant evidence of selection in human populations, and although not enough is known about this SNP in global populations or its function in normal or disease states, it may be one which differentiates Kraepelinean "dementia praecox" from "manic depression". 19,37

The rs701567 SNP in DAOA was not associated with BD diagnosis in this study, contrary to the finding of Fallin et al. ${ }^{16}$ The $\mathrm{G}$ allele was not associated with any phenotype or severity measures; however there was a significant gene-gene interaction with GRIN2B rs 1019385 SNP on the number of hospitalisations for mania. In the South African context, hospitalisation is most often required for emergency treatment of severe episodes. The combination of both ancestral alleles ( $G$ for DAOA and T for GRIN2B) conferred the lowest risk of admission while homozygotes with a derived allele/ancestral allele combination had the highest risk for hospitalisation. Homozygotes for both derived alleles had a relatively low risk of hospitalisation. DAOA activates DAO which determines synaptic glutamate and serine levels, while NMDA receptor function varies according to glutamate levels and GRIN2B genotype. Both DAOA and GRIN2B form part of the phosphoinositide-3 kinase/ AKT-signalling network (PI3K/AKT), involved in the regulation of cell proliferation, growth, development and apoptosis. ${ }^{38,39} \mathrm{We}$ may speculate that heterozygotes have intermediate glutamate function and homozygotes an inflexible regulatory capacity for glutamate load or deficiency states, compromising normal neural development and plasticity.

A major limitation of this study was the relatively small sample size. To avoid this, recent studies have focussed on obtaining large consortia for genetic association tests. An example of this would be the Wellcome Trust Case Control Consortium which investigated the genetic basis of seven common diseases, including BD, using 2000 affected individuals for each disorder and 3000 shared controls. The outcome of this study was that modest genetic effects were able to be detected using larger sample sizes. ${ }^{40}$
The most constraining limitation of the current study is the lack of information about the in vivo and in vitro function of the specific SNPs examined. Future work is needed to determine the functionality of glutamate SNPs in terms of gene expression and physiological correlates, and whether these link to phenotypes for BD. The interaction between DAOA rs701567 and GRIN2B rs 1019385 may be indirect or direct and if direct could be examined with a protein interaction assay such as yeast-2-hybrid screen. Other SNPs in the genes we examined and other genes involved in glutamate metabolism including glutamate receptor, ionotropic, N-methyl D-aspartate l (GRIN1), glutamate receptor, metabotropic l (GRMl) and glutamate receptor, ionotropic, kainate 1 (GRIKl) and their interactions with BD phenotypes should also be explored. Another consideration for future work would be to have greater detail regarding cognitive phenotype. This could be helpful to test (post-hoc) hypotheses related to cognitive function in, for example, controls with GRM3 rs6465084 derived allele homozygotes.

\section{Conclusion}

To our knowledge this is the first study investigating these 3 glutamatergic SNPs and their interactions with BD diagnosis and phenotype. The study provided preliminary evidence that ancestral alleles in the 3 SNPs examined have a protective effect by decreasing the chances of having more severe phenotypes of BD, specifically psychosis and repeat hospital admission. It is possible to speculate that newly derived genes associated with various evolutionary advantages (including perhaps cognitive advantages), may also increase the risk for more severe bipolar disorder. However, findings from this pilot study warrant further research and validation.

\section{Acknowledgements}

We would like to thank Sr Gameda Benefeld for conducting the psychiatric interviews and Ms Alvera Vorster for intellectual input. We acknowledge the funding from the Medical Research Council (MRC), the National Research Foundation (NRF), the University of Cape Town and the Benfara Scholarship.

\section{References}

1. Merikangas KR, Akiskal HS, Angst J, Greenberg PE, Hirschfeld RMA, Petukhova $M$, et al. Lifetime and 12-month prevalence of bipolar spectrum disorder in the National Comorbidity Survey replication. Arch.Gen.Psychiatry 2007;64:543-552.

2. Pini S, de Queiroz V, Pagnin D, Pezawas L, Angst J, Cassano GB, et al. Prevalence and burden of bipolar disorders in European countries. European Neuropsychopharmacology 2005; 15(4):425-434.

3. Müller-Oerlinghausen B, Berghöfer A, Bauer M. Bipolar disorder. The Lancet 2002;359(9302):241-247.

4. Murray CJL, Lopez AD. Global Burden of Disease: A Comprehensive Assessment of Mortality and Morbidity from Diseases, Injuries and Risk Factors in 1990 and Projected to 2020. : Harvard: World Health Organisation; 1996.

5. World Health Organisation. Chapter 2: Burden of Mental and Behavioural Disorders. ; 2001.

6. American Psychiatric Association. Task Force on DSM-IV. DSM-IV. diagnostic and statistical manual of mental disorders. : American Psychiatric Association Washington, DC; 1994.

7. Carter C, Tasha D, Mundo E, Parikh SV, Kennedy JL. Early age at onset as a risk factor for poor outcome of bipolar disorder. J.Psychiatr.Res. 2003;37(4):297-303. 
8. Perlis RH, Miyahara S, Marangell LB, Wisniewski SR, Ostacher M, DelBello MP, et al. Long-term implications of early onset in bipolar disorder: data from the first 1000 participants in the systematic treatment enhancement program for bipolar disorder (STEP-BD). Biol.Psychiatry 2004;55(9):875-881.

9. Green EK, Raybould R, Macgregor S, Hyde S, Young AH, O'Donovan $M C$, et al. Genetic variation of brain-derived neurotrophic factor (BDNF) in bipolar disorder: case-control study of over 3000 individuals from the UK. The British Journal of Psychiatry 2006;188(1):21.

10. Muller DJ, De L. Brain-derived neurotrophic factor (BDNF) gene and rapid-cycling bipolar disorder: family-based association study. The British Journal of Psychiatry 2006;189(4):317.

11. Vincze I, Perroud N, Buresi C, Baud P, Bellivier F, Etain B, et al. Association between brain-derived neurotrophic factor gene and a severe form of bipolar disorder, but no interaction with the serotonin transporter gene. Bipolar Disord. 2008; 10(5):580-587.

12. Palomino A, González-Pinto A, Aldama A, González-Gómez C, Mosquera F, González-García G, et al. Decreased levels of plasma glutamate in patients with first-episode schizophrenia and bipolar disorder. Schizophr.Res. 2007;95(1-3):174-178.

13. Manji HK, Quiroz JA, Payne JL, Singh J, Lopes BP, Viegas JS, et al. The underlying neurobiology of bipolar disorder. World Psychiatry 2003;2(3):136.

14. Karkanias NB, Papke RL. Subtype-specific effects of lithium on glutamate receptor function. J.Neurophysiol. 1999;81 (4):1506-1512.

15. Paddock S, Laje G, Charney D, Rush AJ, Wilson AF, Sorant AJM, et al. Association of GRIK4 with outcome of antidepressant treatment in the STAR* D cohort. Am.J.Psychiatry 2007;164(8):1181.

16. Fallin MD, Lasseter VK, Avramopoulos D, Nicodemus KK, Wolyniec PS, McGrath JA, et al. Bipolar I disorder and schizophrenia: a 440-singlenucleotide polymorphism screen of 64 candidate genes among Ashkenazi Jewish case-parent trios. The American Journal of Human Genetics 2005;77(6):918-936.

17. Lyon L, Kew JNC, Corti C, Harrison PJ, Burnet PWJ. Altered hippocampal expression of glutamate receptors and transporters in GRM2 and GRM3 knockout mice. Synapse 2008;62(11).

18. Choudary P, Molnar M, Evans S, Tomita H, Li J, Vawter M, et al. Altered cortical glutamatergic and GABAergic signal transmission with glial involvement in depression. Proceedings of the National Academy of Sciences 2005;102(43):15653-15658.

19. Crespi B, Summers K, Dorus S. Adaptive evolution of genes underlying schizophrenia. Proceedings of the Royal Society B: Biological Sciences 2007;274(1627):2801-2810

20. Egan MF, Straub RE, Goldberg TE, Yakub I, Callicott JH, Hariri AR, et al. Variation in GRM3 affects cognition, prefrontal glutamate, and risk for schizophrenia. Proceedings of the National Academy of Sciences 2004;101(34):12604-12609.

21. Tang YP, Wang H, Feng R, Kyin M, Tsien J. Differential effects of enrichment on learning and memory function in NR2B transgenic mice. Neuropharmacology 2001;41 (6):779-790.

22. Martucci L, Wong AHC, De Luca V, Likhodi O, Wong GWH, King N, et al. N-methyl-d-aspartate receptor NR2B subunit gene GRIN2B in schizophrenia and bipolar disorder: Polymorphisms and mRNA levels Schizophr.Res. 2006;84(2-3):214-221

23. Avramopoulos D, Lasseter VK, Fallin MD, Wolyniec PS, McGrath JA, Nestadt G, et al. Stage II follow-up on a linkage scan for bipolar disorder in the Ashkenazim provides suggestive evidence for chromosome 12p and the GRIN2B gene. Genetics in Medicine 2007:9(11):745.

24. Miyatake R, Furukawa A, Suwaki H. Identification of a novel variant of the human NR2B gene promoter region and its possible association with schizophrenia. Mol.Psychiatry 2002:7:1101-1106.

25. Chumakov I, Blumenfeld M, Guerassimenko O, Cavarec L, Palicio M, Abderrahim $H$, et al. Genetic and physiological data implicating the new human gene G72 and the gene for D-amino acid oxidase in schizophrenia. Proceedings of the National Academy of Sciences 2002;99(21):13675-13680.

26. Hashimoto K, Engberg G, Shimizu E, Nordin C, Lindström LH, Iyo M. Reduced D-serine to total serine ratio in the cerebrospinal fluid of drug naive schizophrenic patients. Progress in Neuropsychopharmacology \& Biological Psychiatry 2005;29(5):767769.

27. Hattori E, Liu C, Badner JA, Bonner TI, Christian SL, Maheshwari M, et al. Polymorphisms at the G72/G30 gene locus, on 13q33, are associated with bipolar disorder in two independent pedigree series. The American Journal of Human Genetics 2003;72(5):1131-1140.

28. Chen Y, Akula N, Detera-Wadleigh S, Schulze T, Thomas J, Potash J, et al. Findings in an independent sample support an association between bipolar affective disorder and the G72/G30 locus on chromosome 13q33. Mol.Psychiatry 2004;9(1):87-92.

29. Addington AM, Gornick M, Sporn AL, Gogtay N, Greenstein D, Lenane $M$, et al. Polymorphisms in the 13q33. 2 gene G72/G30 are associated with childhood-onset schizophrenia and psychosis not otherwise specified. Biol.Psychiatry 2004;55(10):976-980.

30. Korostishevsky M, Kaganovich M, Cholostoy A, Ashkenazi M, RatnerY, Dahary D, et al. Is the G72/G30 locus associated with schizophrenia? Single nucleotide polymorphisms, haplotypes, and gene expression analysis. Biol.Psychiatry 2004;56(3):169-176.

31. Schumacher J, Jamra RA, Freudenberg J, Becker T, Ohlraun S, Otte A, et al. Examination of G72 and D-amino-acid oxidase as genetic risk factors for schizophrenia and bipolar affective disorder. Mol.Psychiatry 2004; 9(2):203-207.

32. Wang X, He G, Gu N, Yang J, Tang J, Chen Q, et al. Association of G72/G30 with schizophrenia in the Chinese population. Biochem.Biophys. Res. Commun. 2004;31 9(4):1281-1286

33. Le-Niculescu H, Patel S, Bhat M, Kuczenski R, Faraone S, Tsuang M, et al. Convergent functional genomics of genome-wide association data for bipolar disorder: Comprehensive identification of candidate genes, pathways and mechanisms. 2008.

34. Norton N, Williams HJ, Dwyer S, Ivanov D, Preece AC, Gerrish A, et al. No evidence for association between polymorphisms in GRM3 and schizophrenia. BMC Psychiatry 2005 May 13;5:23.

35. Tochigi M, Suga M, Ohashi J, Otowa T, Yamasue H, Kasai K, et al. No association between the metabotropic glutamate receptor type 3 gene (GRM3) and schizophrenia in a Japanese population. Schizophr.Res. 2006;88(1-3):260-264.

36. Diller KC, Gilbert WA, Kocher TD. Selective sweeps in the human genome: A starting point for identifying genetic differences between modern humans and chimpanzees. Mol.Biol.Evol. 2002;19(12):23422345.

37. Kraepelin E. Über Sprachstörungen im Traume. : Verlag von Wilhelm Engelmann; 1906.

38. Carter C. Multiple genes and factors associated with bipolar disorder converge on growth factor and stress activated kinase pathways controlling translation initiation: implications for oligodendrocyte viability. Neurochem.Int. 2007;50(3):461-490.

39. Vara JÁF, Casado E, de Castro J, Cejas P, Belda-Iniesta C, GonzálezBarón M. PI3K/Akt signalling pathway and cancer. Cancer Treat.Rev. 2004;30(2): 193-204.

40. Rahman SN, Stratton M. Genome-wide association study of 14,000 cases of seven common diseases and 3,000 shared controls. Nature 2007;447(7145):661-678. 